\title{
Grimms Wissenschaft, Grimms Deutsch
}

In seiner Deutschen Grammatik von 1819, im Kapitel zu den Konjugationen, kommt Jacob Grimm auf die Frage der sprachwissenschaftlichen Terminologie zu sprechen. Es geht um die richtige Bezeichnung für die Verben, die andere als ,unregelmäßig‘ bezeichnen - ein Begriff, den Grimm mit einiger Emphase als irreführend zurückweist. Die fraglichen Verben seien alles andere als unregelmäßig. Vielmehr zeigten sie - trotz oder vielmehr in der Vielfalt ihrer Formen - eine ,Regel', die wie ein roter Faden die ganze Wortbildung der germanischen Sprachen durchziehe. Um diese Regel zu erkennen, bedürfe es eines ganz anderen Vokabulars: statt von ,unregelmäßig‘ und ,regelmäßig‘ solle von ,stark‘ und ,schwach` die Rede sein; statt von ,Anomalie‘ von ,Ablaut':

\begin{abstract}
Ablaut. Die Veränderung des Wurzellauts im Präteritum ist die Seele der starken, d. h. der eigentlichen ältesten Conjugationsform und eine durch alle deutsche Sprachen ziehende Grundeigenthümlichkeit, die sie von den meisten andern vortheilhaft auszeichnet. - Man hat die ablautenden Verba sehr verkehrt als unregelmäßige, anomale Fälle behandelt, in welche kein System zu bringen sey und sie den schwachen Wörtern nachgesetzt. Es offenbart sich aber gerade in ihnen die tiefsinnige Ordnung des Sprachgeistes, der, was er im Einzelnen zu wirken scheint, im Ganzen ausgerichtet hat. Die Regel, die wir hier bei der Conjugation erkennen, thut sich wunderbar in den Verhältnissen der ganzen Wortbildung überhaupt hervor. ${ }^{1}$
\end{abstract}

Unter ,Ablaut‘ versteht Grimm also nicht nur den charakteristischen Vokalwechsel der ,starken' Verben - singen, sang, gesungen - sondern ein grundlegendes Prinzip verschiedener Wortbildungsprozesse, auch beim Substantiv - Binde, Band, Bund. Es handelt sich um ein, wie es auch in dem von Grimm selbst verfassten Artikel Ablaut im Deutschen Wörterbuch heißt, ,edles und ihr wesentliches vermögen der deutschen sprache“² - das von der üblichen Terminologie geradezu verstellt wird. Die ablautenden Verba sind also nicht unregelmäßig, sie sind stark.

,Ablaut', ,Rückumlaut', ,Brechung', ,Anlaut', ,Inlaut' und ,Auslaut', ,stark und ,schwach': das sind einige der Fachausdrücke, die Jacob Grimm entweder neu geprägt oder in die Sprache der Sprachwissenschaft allererst eingeführt hat. ${ }^{3}$ In die Sprache, wohlgemerkt, der sich erst in seiner Zeit herausbildenden historischen und vergleichenden Wissenschaft von der Sprache, einer Disziplin, zu deren

1 Grimm 1819/1995: 546f.

2 Grimm \& Grimm (1854-1971), Bd. 1: 69. Nachweise im Folgenden mit Sigle DWB und Angabe der Band- und Spaltenzahl direkt im Text.

3 Vgl. Ginschel 1956: 47. Zur Vorgeschichte der Termini ,Umlaut‘ und ,Ablaut‘ vgl. Ising 1956.

Ә Open Access. (C 2019 David Martyn, publiziert von De Gruyter. (cc))BY-NC-ND Dieses Werk ist lizenziert unter der Creative Commons Attribution-NonCommercial-NoDerivatives 4.0 Lizenz.

https://doi.org/10.1515/9783110676631-009 
Gründern neben Franz Bopp und Rasmus Kristian Rask nicht zuletzt Jacob Grimm selbst gehört. Grimm steht, so scheint es, an just der Stelle, an der sich die beiden uns interessierenden Prozesse kreuzen: einerseits die Ausdifferenzierung der ,Geisteswissenschaften“ um 1800, in der insbesondere die Sprachwissenschaft eine Vorreiterrolle einnimmt; und andererseits die Ablösung des Lateinischen durch die verschiedenen Volkssprachen, insbesondere das Deutsche, als Medium wissenschaftlicher Kommunikation. ${ }^{4}$ Was der Sprachforscher Grimm mitbegründete, war eine Wissenschaft; und die Sprache, in der er diese Wissenschaft betrieb, war Deutsch. So jedenfalls scheint es. Denn schaut man sich die Verhältnisse etwas genauer an, stellen sich gewisse wenn nicht Zweifel, dann Komplikationen ein Komplikationen, die, wie ich meine, für unsere Suche nach der Wissenschaftssprache ,Deutsch“ von Interesse sein sollten. Es fragt sich nämlich, was genau unter ,Wissenschaft‘ verstanden werden kann, wenn Grimms Sprachwissenschaft zu ihr gehören soll; zweitens aber, ob die Sprache, in der Grimm seine Sprachwissenschaft betrieb, überhaupt ,Deutsch“ war und wenn ja, in welchem Sinne. Der Fall Grimm bietet sich an, um sowohl den Begriff der Wissenschaft als auch den des Deutschen auf produktive Weise historisch neu zu reflektieren. Ich wende mich zuerst der Frage der Wissenschaft und der Wissenschaftssprache zu, dann, etwas ausführlicher und mit einem kurzen Exkurs über die sogenannte Sprachinhaltsforschung Jost Triers und Leo Weisgerbers, die zu Vergleichszwecken herangezogen wird, der Frage nach dem ,Deutsch“ dieser Sprache.

\section{Grimms Wissenschaft}

Folgt man der Archäologie der Humanwissenschaften, wie sie Michel Foucault in der Ordnung der Dinge dargelegt hat, dann ist für die Human- und zumal die Geisteswissenschaften seit ihrem Entstehen eine bestimmte Aporie charakteristisch. Die Entdeckung der Positivitäten in den Humanwissenschaften in der Zeit nach 1800 lässt eine problematische Kluft entstehen - zwischen den empirischen Tatsachen, die durch die positive Wissenschaft in eine objektivierende Distanz gerückt werden, und dem sie beobachtenden Erkenntnissubjekt ,Mensch“, dessen eigentliches Sein ihm nunmehr auf der Seite jener stummen Empirie unheimlich entgegenblickt. Es tut sich die Spaltung auf, aus der jene „paradoxe Gestalt“ des Menschen von nun an bestehen wird: als „empirisch-transzendentale Dublette“

4 Vgl. dazu nach wie vor Pörksen 1986: 42-71. Für eine kultur- und wissenschaftstheoretisch fundierte Aufarbeitung der ganzen Problematik seit Paracelsus vgl. Pörksen 1994. 
steht der Mensch dem „stummen Horizont“ der empirischen Inhalte, der „sandigen Weite des Nicht-Denkens“ gegenüber, das sein eigenes Denken zugleich ermöglicht und überbordet. ${ }^{5}$ Besonders in der neuen historischen Sprachwissenschaft, so Foucault, werde das Problem akut. War die Sprache im klassischen Zeitalter, in der Zeit der ,allgemeinen Grammatik‘, noch unmittelbar an die Formen des Denkens geknüpft, erhält sie jetzt, im Zeitalter von Rask, Bopp und nicht zuletzt Grimm, „plötzlich ein eigenes Sein“6 - und erschüttert damit das Selbstverständnis des zoon logikon. „Wie kann [der Mensch] das Subjekt einer Sprache sein, die seit Jahrtausenden ohne ihn gebildet worden ist, deren System ihm entgeht, deren Bedeutung [...] in den Wörtern ruht, [...] innerhalb deren er [...] sein Denken plazieren muß?" ${ }^{7}$ lautet die an sich unlösbare Frage, die sich erhebt, sobald die vergleichende Grammatik die Sprache zu einem „Erkenntnisgegenstand unter

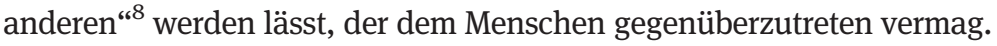

Bereits 1979, in seiner nach wie vor einschlägigen Studie zu Jacob Grimm, hat Ulrich Wyss allerdings überzeugend darlegen können, dass Grimms Wissenschaft in dieses Schema durchaus nicht passt. Denn der Grimm'sche Ansatz, so Wyss, lasse die Spaltung von erkennendem Subjekt und Forschungsgegenstand gar nicht erst aufkommen: dadurch nämlich, dass er das Subjekt dem Erkenntnisobjekt gleichsam ausliefere. Um es zuzuspitzen: Grimm redet nicht über die Sprache, sondern mit ihr. ${ }^{9}$

Was das genauer heißt, könne man etwa daran sehen, dass bei Grimm die verschiedenen sprachwissenschaftlichen Genres immer wieder durcheinandergeraten. So beginnt das Deutsche Wörterbuch (ab 1854) mit einem Artikel über den Buchstaben , $\mathrm{A}^{\star}$ - an sich nichts Ungewöhnliches, auch Adelung hatte so begonnen -, der aber sofort in eine lange Erklärung der Lautgesetze der germanischen Sprachen, nicht zuletzt des Umlauts, ausufert: alles Dinge, die man nicht im Wörterbuch, sondern in einer Grammatik erwarten würde. Die Deutsche Grammatik wiederum (ab 1819) kann die Funktion des Ablauts in der Wortbildung, an sich eine reine Formfrage, nicht ohne ausgedehnte Spekulationen über die Etymologien einzelner Wörter behandeln, die ja eigentlich Sache der Lexikographie wären: ${ }^{10}$ so etwa bei der Behandlung des ablautenden Vokalwechsels vom Präsens zum Präteritum, bei der Grimm sogleich auffällt, dass der Ablaut mehr als nur die Zeitstufe ändert. In zahlreichen Fällen werde nämlich die „urbedeutung“ der Wurzel im ablautenden

5 Foucault 1971: 389.

6 Ebd.: 390.

7 Ebd.

8 Ebd.: 360.

9 Vgl. Wyss 1979: 167.

10 Vgl. dazu und zum Folgenden ebd.: 148-154. 
Präteritum sowie in den vom Präteritum abgeleiteten Substantiva und Adjektiva ,geschwächt, umgedreht', ja ,negiert‘ oder gar ,geleugnet‘: „Wörter, welche ein schließen, decken, voll sein ausdrücken, pflegen im praet. zuweilen das offene, hohle zu bezeichnen, wie der schlüßel zu, aber auch wieder aufmacht, die thüre deckt und öffnet“. ${ }^{11}$ Nach einer ähnlichen Logik zieht Grimm ,Schwäche` zu althochdeutsch „swecchan“, ,stinken“, als „etwas, das ausgerochen hat, verwelkt ist“ ${ }^{12}$ „Mich dünkt,“ überlegt Grimm nach diesen detaillierten Beobachtungen, „wie der erste ablaut schon den begriff des urlauts mindere, und aus heller gegenwart in stillere vergangenheit setze, daß der zweite ablaut die bedeutung wiederum noch mehr abstumpfe, entstelle und gegen jenen gehalten abstracter mache“.${ }^{13}$ Kaum hat Grimm also begonnen, den sezierenden Blick des Grammatikers auf die Sprache zu richten, versenkt er sich in den Inhalt, ,verfällt‘ gleichsam dem ,Stoff‘. Positiv gewendet: statt die Sprache den analytischen Kategorien der Sprachwissenschaft zu unterwerfen, schreibt er einen Text, der die Bewegungen, Abwandlungen, Sprünge, die er der Sprache attestiert, selbst vollzieht. Das erkennende Subjekt steht den Positivitäten der Sprache nicht gegenüber, sondern es wohnt mittendrin.

Dies zeigt sich nun auch, so ließe sich an Wyss' Argumentation anknüpfen, an Grimms Wissenschaftssprache. $\mathrm{Zu}$ ihren Eigentümlichkeiten gehört, dass die ,technischen“ Begriffe sich kaum als ,Termini‘ herauslösen lassen, so innig sind sie mit dem übrigen Diskurs verwoben. So ist etwa nicht nur von ,Ablaut ' die Rede, sondern von ,ablautenden Verben' oder von Wurzeln, die ,ablauten`. ${ }^{14}$ ,Schwach“ ist zwar eine Kategorie zur Einteilung der Verben, aber als ganz ,normales' Adjektiv wird das Wort auch weiterhin verwendet - und zwar so, dass die beiläufigen Verwendungen durchaus mit den ,technischen' in einer Verbindung stehen, die Auslegungssache bleibt. Wenn es etwa bei der Unterscheidung zwischen ,Umlaut‘ und ,Ablaut' heißt, der Umlaut bestehe in der „Trübung oder Schwächung des reinen Vocal[s]“, während beim Ablaut „der Vocal in einen andern, aber ebenfalls reinen, ab- und überspringt“, ${ }^{15}$ lässt dieser Wortgebrauch nicht ohne Grund an die ,Schwäche“ der schwachen Flexion denken, die des starken ,Vokalsprungs“ von einem reinen Vokal in den anderen entbehrt. Vokale können nicht nur ,geschwächt‘, sondern auch „,verdünnt“ oder „getrübt“ werden," was durchaus etwas miteinander zu tun zu haben scheint, ohne dass sich diese

\footnotetext{
11 Grimm 1878/1989: 77.

12 Ebd.: 76.

13 Ebd.: 79.

14 Vgl. etwa Grimm 1819/1995: 555.

15 Ebd.: $542 \mathrm{f}$.

16 Grimm 1840/1995: 554.
} 
Bezeichnungen - Grimms wiederholten Bemühungen zum Trotz - genau festlegen ließen. Eine Sprache mit intaktem, ,starkem‘ Konjugations- und Deklinationssystem kann bei dem Vergleich mit anders strukturierten Sprachen als ,kräftig“ bezeichnet werden - und zwar durchaus wegen der semantischen Nähe der beiden Adjektive. ${ }^{17}$ Wie diese Belege zeigen, behalten die ,Fachbegriffe‘ in Grimms Prosa ihre volle morphologische und semantische Anschlussfähigkeit, sie erstarren nie zu termini technici, sondern bleiben, um es mit einem Grimmwort auszudrücken, ,lebendig‘. Man versteht, warum im Jahre 1956 ein Bericht aus der Arbeit an einem historischen Wörterbuch der sprachwissenschaftlichen Terminologie, das nie fertiggestellt wurde, zu dem Schluss kommen konnte: „Grimms „Begriffe“ lassen sich nur historisch nachzeichnen, nicht definieren“. ${ }^{18}$ Das aber bedeutet, dass es bei Grimm nie zur Ablösung einer beschreibenden oder analytischen Metasprache kommt, mit der die Sprache von ,außerhalb` als Gegenstand behandelt werden könnte. Die Sprache der Wissenschaft bleibt offen gegenüber der Sprache, die sie untersuchen will. Auch das ebnet die Kluft ein, die sonst zwischen Erkenntnismittel und Gegenstand aufklafft.

Damit aber erhebt sich zugleich die Frage, wie es mit Grimms Wissenschaft letztlich steht. Wenn bei Grimm das erkennende Subjekt keinen objektivierenden Abstand von seinem Gegenstand einhält; wenn sich keine Metasprache von der Objektsprache abspaltet: Kann dann überhaupt von Wissenschaft die Rede sein - und wenn ja, in welchem Sinne? Die Haltung seitens der Germanistik gegenüber Grimm ist bekannt: Er sei zwar der Gründer der Disziplin, zugleich aber gänzlich undiszipliniert, ein Stoffhuber, der das Angesammelte häuft, ohne es wissenschaftlich auszuwerten. ${ }^{19}$ Gemeint ist damit wohl auch: ohne den nötigen Abstand zu seinem Material einzuhalten, um es wissenschaftlich aufzubereiten. Dieses gängige Urteil über Grimms fehlende Wissenschaftlichkeit, über seine ,Romantik', lässt sich durchaus nachvollziehen. Beim Lesen der Deutschen Grammatik, der von Grimm verfassten Artikel im Deutschen Wörterbuch, der Geschichte der deutschen Sprache wird man einerseits von der Fülle des Materials überwältigt, andererseits stets daran erinnert - durch Exkurse, durch die eigenwillige Anordnung, durch subjektive Bewertungsbekundungen -, dass hier ein ganz bestimmter Autor am Werke ist.

17 „Die starke Conjugationsform verdient so zu heißen: 1) weil sie lauter einfache kräftige Wurzeln enthält, die schwache hingegen meistens Ableitungen [...].“ (Grimm 1819/1995: 558) Zur Metaphorik von ,stark‘ und ,schwach“ und ihrer Rolle als ,Kraft‘ in Grimms Konzept der Sprache vgl. Krapf 1993: 57-66.

18 Ginschel 1956: 102.

19 Vgl. Wyss 1979: 1. 
Andererseits aber hat die historische Epistemologie - man denke nur an Fleck, Canguilhem, Bachelard, Rheinberger - den Begriff der ,Wissenschaft", auf dem dieses Urteil beruht, als historisch kurzsichtig entlarvt. Der Anspruch der Wissenschaften, ihre Gegenstände stets in objektivierender Ferne zu halten, ist erstens einem ganz bestimmten Kapitel in der Geschichte des Wissens eigen und zweitens nie wirklich eingehalten worden. ${ }^{20}$ Vielmehr haben wir es im Falle der ,Wissenschaften' mit einer Wissensart unter anderen zu tun, die erst im Rahmen einer historischen Epistemologie angemessen eingeschätzt werden kann. Anstatt zu fragen, ob Grimms linguistische Forschung ,wissenschaftlich“ war - nämlich im Sinne eines unhistorischen Begriffs von ,Wissenschaft ${ }^{\text {* }}$, ist man besser beraten, die Frage umzukehren und zu eruieren, was uns Grimms Philologie über den Begriff der Wissenschaft selbst und seine historischen Kontingenzen zeigen kann. Und dann ließe sich sagen, dass die aporetische Spaltung des erkennenden Subjekts von seinem Gegenstand, die bei Foucault als symptomatisch für die Humanwissenschaften schlechthin figuriert, nicht ohne Ausnahme gilt. Denn dass Grimm nur Material gehäuft habe, ohne es wissenschaftlich auszuwerten - dass er die Dinge entdeckt habe, nicht aber die Ordnung -, ist eine Fehleinschätzung. Die Studie von Wyss zeigt in extenso, welches Wissen die Philologie Grimms bereitstellt.

Ja noch mehr: Grimms Wissenschaftssprache, darauf möchte ich im Anschluss an Wyss` Ergebnisse hinweisen, vermag ihre Masse an Material besonders elegant zu ordnen, gerade weil sie keinen Abstand zu ihrem Gegenstand hält. Gerade in ihrer Offenheit und Flexibilität vermögen Grimms Begrifflichkeiten Phänomene zusammenzufassen, die sonst einzeln behandelt werden müssten: etwa indem die ganze Flexion, sowohl der Verben als auch der Substantive und Adjektive, nach der Unterscheidung von ,stark' und ,schwach“ dargestellt wird. ${ }^{21}$ Ähnlich fungiert der berühmte ,Urbegriff‘ in den Artikeln des Deutschen Wörterbuchs, jene nirgends belegte, sinnlich-konkrete Urbedeutung, die aus dem Belegmaterial konjiziert wird und der Geschichte eines jeden Wortes nicht nur zugrunde liegen, sondern über ihre ganze Länge unausgesprochen wirksam bleiben soll. ${ }^{22}$ Ganz gleich, wie man es mit der historischen Plausibilität dieser Etymologien halten

20 Wovon man sich etwa durch Rheinbergers eindrucksvolle Rekonstruktion der Entdeckung der Proteinsynthese durch den Harvard-Onkologen Paul C. Zamecnik überzeugen lassen kann, vgl. Rheinberger 1997.

21 Vgl. Grimm 1880/1999: 652.

22 „Hinter allen abgezognen bedeutungen des worts liegt eine sinnliche und anschauliche auf dem grund, die bei seiner findung die erste und ursprüngliche war. es ist sein leiblicher bestandtheil, oft geistig überdeckt, erstreckt und verflüchtigt, alle worterklärung, wenn sie gedeihen soll, musz ihn ermitteln und entfalten.“ (DWB 1: xlv) Vgl. dazu Wyss 1979: $179 \mathrm{f}$. 
mag: Sie erlauben eine äußerst eingängige Darstellung der Entwicklung der Wortbedeutungen. Spätere historische Wörterbücher, etwa Daniel Sanders Wörterbuch der deutschen Sprache (ab 1876), gliedern die Bedeutungen viel methodischer nach dem aristotelischen Prinzip von genus proximum und differentia specifica. ${ }^{23}$ Damit ergibt sich eine zwar übersichtliche, aber künstliche Aufteilung des Bedeutungsfelds, deren Form nur erzwungenermaßen zum Inhalt passt. Denn Wörter geschichtlich entstandener Sprachen verhalten sich eben nicht wie die Termini einer Taxonomie. Ein Wörterbuch, das so vorgeht, täuscht eine künstliche Übersichtlichkeit vor, die mit der Geschichte der lemmatisierten Wörter wenig zu tun hat - und das in Artikeln, die sich als Texte nicht lesen lassen; man behält von ihnen nur wenig. Grimms Artikel dagegen sind Narrative, sie erzählen gleichsam Biographien der Wörter. Die ganze heterogene Masse der Positivitäten kommt in ihnen nicht weniger zur Darstellung, aber in einer Form, die es ermöglicht, sie auch aufzunehmen.

Gerade darin läge also der Vorteil einer Sprachwissenschaft, die ihren Gegenstand nicht einer von ihr vorab festgelegten Ordnung unterwirft, sondern sich ihm eben ,ausliefert‘. Im Fall von Jacob Grimm hätten wir somit einen Beleg für eine Spielart der Wissenschaft - Goethes Morphologie wäre ein anderer ${ }^{24}$-, deren Leistung sich gerade der Annäherung der Form der wissenschaftlichen Darstellung an deren Gegenstand verdankt.

\section{Grimms Deutsch}

Es sieht also ganz danach aus, als läge mit Grimms Philologie tatsächlich ein Paradebeispiel dafür vor, was Deutsch als Wissenschaftssprache in der Zeit um 1800 leisten konnte. Der Befund, dass bei Grimm Meta- und Objektsprache ineinander übergehen, schiene mit diesem Ergebnis nicht nur in Einklang zu stehen, sondern ihm eine noch tiefere Signifikanz zu verleihen. Denn die Objektsprache ist das Deutsche selber. Wenn Grimms Wissenschaftssprache gegenüber der deutschen Sprache als ihrem Gegenstand offen bleibt, wenn bei ihm gar Meta- und Objektsprache deckungsgleich sind, dann ist Grimms Wissenschaftssprache nichts mehr und nichts weniger als Deutsch - und zwar mit einer Prägnanz, die andere Wissenschaften, die in deutscher Sprache betrieben werden, nicht ohne Weiteres für sich beanspruchen können. In der deutschen Philologie wie sonst nirgendwo besteht die Möglichkeit, so scheint es, Deutsch in wissenschaftlicher Absicht auf

23 Vgl. dazu Reichmann 1991.

24 Vgl. Geulen 2016; vgl. ferner Krapf 1993: 13-15. 
sich selbst anzuwenden: als Objekt- und als Wissenschaftssprache zugleich. Und demnach hätte Grimms Philologie diese Möglichkeit voll ausgeschöpft: indem sie an keiner Stelle aufhörte, die Sprache zu sprechen, die ihr Gegenstand war; indem sie es nie dazu kommen ließ, dass sich eine Fachsprache von der Sprache, um die es ihr ging - nämlich der ,lebendigen“ deutschen Sprache, wie sie sich geschichtlich entwickelt - abspaltet. ,Ablaut', ,stark', ,schwach': das wären dann bei Grimm alles Fachausdrücke und zugleich deutsche Wörter, Wörter, die im vollen Sinne des Worts der deutschen Sprache angehören.

Und so ist es auch - allerdings mit einem kleinen, aber wesentlichen Vorbehalt. Denn es fragt sich, was für ein ,Deutsch“ das ist, das Grimm schreibt. Man erwäge den Status der von mir hervorgehobenen Wörter in den folgenden Zitaten, alle aus dem Vorwort zum Deutschen Wörterbuch:

1. zur forschung über den verhalt der alten, verschollenen sprache fühlen wenige sich berufen [...]. (DWB 1: vii)

2. jedermann weisz es, wie viel beschwer in slavischen wörterbüchern die mannigfaltigkeit einiger bezeichnungen auch für die alphabetische folge macht [...]. (DWB 1: xi)

3. den lange verhaltnen tadel [über Adelungs Wörterbuch] sprach endlich Voss treffend und bitter aus, dennoch ungerecht, weil ihm die anerkennung dessen abgieng, was in engem, freiwillig gestecktem befang mit reichem, allen nützendem ertrag geerntet worden war. (DWB 1: xxiv)

4. [...] grammatische[ ] entdeckungen [...] aus sparsam flieszenden fast versiegenden althochdeutschen und gothischen quellen, die uns unserer zunge älteste und gefügeste regel kund thaten. (DWB 1: iv)

Verhalt, Beschwer, Befang, gefügest: Grimms Wortgebrauch beschränkt sich, wie man sieht, nicht auf den gebräuchlichen Wortbestand des Neuhochdeutschen. Es ist oft gesagt worden, dass Grimms sprachgeschichtliche Kenntnisse in seine eigene Sprache hineingeflossen sind, und dem ist auch so. Das Adjektiv „gefüge“ zum Beispiel (Zitat 4), im Sinne von ,fügsam‘, ,passend', ,geschickt‘, ist zu Grimms Zeiten - wie uns das Deutsche Wörterbuch belehrt - „wie im aussterben begriffen“, wäre aber, so der von Grimms Nachfolger verfasste Artikel weiter, „gut wieder zu beleben“ (DWB 4.1.2: 2164). „[B]eschwer“ für ,Beschwerlichkeit“ (Zitat 2) und „verhalten“ im Sinne von ,zurückhalten“ (Zitat 3) sind nicht ganz so altertümlich, zehren aber eindeutig von altem Wortbestand. Anders verhält es sich allerdings in den beiden anderen Fällen: Das Wort „verhalt“ (Zitat 1) erklärt das Deutsche Wörterbuch - ist ,in der älteren Sprache nicht nachzuweisen“ (DWB 12.1: 507); und „befang“ (Zitat 3), im Althochdeutschen im Sinne von ,Einhegung“ nachgewiesen, wird an dieser Stelle, wo man sonst ,Gebiet“ erwarten würde - zumal, da Grimm diesen gebräuchlicheren Ausdruck sonst selber 
benutzt (DWB 1: xiv) -, in einem ganz neuen Sinne verwendet. „[V]erhalt“ und „befang“ sind sowohl alt als auch neu: es sind Neologismen, könnte man sagen, wie sie nach Grimms Meinung die deutsche Sprache seit jeher hätte bilden können. Man hat bereits 1869, sechs Jahre nach seinem Tod, in der ersten ausführlichen Behandlung seiner Sprache, nachgewiesen, dass Grimm an die Stelle von Abstrakta, die gewöhnlich durch die Anfügung einer Nachsilbe wie ,-ung“ oder ,-nis‘ gebildet werden, Neuableitungen von Verben setzt, insbesondere, wie zu erwarten, von ,starken“ oder ,ablautenden‘. So schreibt er etwa „eintrag“ für ,Eintragung“, „übertrag“ für ,Übertragung“, „verschub“ für ,Verschiebung“. ${ }^{25}$ Bezeichnender noch für seine Sprache sind allerdings Wortbildungen, für die es keine genaue Entsprechung zu geben scheint: etwa wo er „entsprung“ gebraucht für die Entstehung eines Lauts aus einem anderen („der entsprung dieser endung $-\mathrm{e}$ aus altem $\left.-\mathrm{u}^{\text {“ }}\right){ }^{26}$,[E]ntsprung“ ist nicht dasselbe wie ,Entstehen“ - sondern, möchte man sagen, „ein glücklich[er] wurf durch kurze und kühne beschreibung der sache“. ${ }^{27}$

In welchem Sinne nun sind diese Neubildungen, die aber zugleich Altbildungen sind, ,Deutsch‘? Grimm selbst würde sicherlich sagen: in einem besonders tiefen Sinne; sie sind Bildungen im Geiste des Deutschen, aus dem deutschen ,Sprachgeist', sofern er in Grimms Feder fortlebt. Mit diesem Sprachgeist aber hat es eine besondere Bewandtnis. Denn offensichtlich werden nur diejenigen von diesem Sprachgeist beseelt, die, wie Grimm, ausgedehnte Kenntnisse der Geschichte der deutschen Sprache besitzen. Die Deutschen selber neigen nämlich eher dazu, wie Grimm in seiner Abhandlung Über das Pedantische in der Deutschen Sprache klar macht, die Sprache durch allerlei Mittel $\mathrm{zu}$ erschweren - etwa durch die vielen, Grimm unerträglichen Neubildungen von Verben auf ,-ieren' -, als sie wie Grimm durch kühne Wortschöpfungen zu vereinfachen, gleichsam zu entschlacken. Um wie Grimm zu schreiben, muss man Philologe sein. Die Sprache, in der er seine Philologie betrieb, ist somit zwar dieselbe, die in ihr erforscht wird - aber sie ist auch deren Ergebnis. Anders gesagt: Grimm untersucht die Sprache, die er zu deren Beschreibung zugleich entdeckt und erfindet. Das auf sich selbst angewandte und aus sich selbst entwickelte Deutsch, das er schrieb und beschrieb, ist ein neues Gebilde.

25 Andresen 1968: 104. Zu Grimms Sprache vgl. ferner Simon 1929 und Wyss 1979: 182-188.

26 Grimm 1870/1989: 635.

27 So Grimms Charakterisierung der wortstiftenden Arbeit des ,Sprachgeistes', Grimm 1878/ 1989: 81. - Zum Begriff des ,Sprachgeistes‘ bei Grimm vgl. Herrlich 1998: insb. 74f., 154; im Kontext des deutschen Sprachnationalismus, in dem Grimm eine zeituntypisch von Fremdenhass freie Rolle zukomme, vgl. Stukenbrock 2005: 318. 
Das wird umso deutlicher, wenn man Grimms besonderes Deutsch mit dem vergleicht, das andere deutsche Sprachwissenschaftler nach ihm gepflegt haben. Gemeint ist die bereits erwähnte ,Sprachinhaltsforschung', die ab den 1930er Jahren von Leo Weisgerber, Jost Trier und anderen entwickelt wurde und nach dem Zweiten Weltkrieg zum führenden Paradigma der bundesdeutschen Sprachwissenschaft avancierte. Sie bietet sich deswegen als Vergleichsfall an, weil sich in ihr die Wende zur Objektivierung der Sprache, die die deutsche Sprachwissenschaft nach Grimm vollzog, wieder umkehrt. Hatte die deutsche Sprachwissenschaft ab der Mitte des 19. Jahrhunderts eine „Neubegründung im Zeichen der Naturwissenschaft“"28 erlebt, eine positivistische Wende, die für solch ,romantische“ Abstraktionen wie Volks- oder Sprachgeist keinen Gebrauch mehr sah, so bildete sich ab der Jahrhundertwende eine antipositivistische Gegenreaktion, die die Sprache wieder als kulturelles Gebilde in den Blick nahm. Zu diesen antipositivistischen Bestrebungen gehörte die Arbeit von Leo Weisgerber. ${ }^{29}$ Bereits mit Weisgerbers erster programmatischer Schrift Muttersprache und Geistesbildung (1929) forderten wieder Begriffe ihr Recht, die in der positivistischen Zeit keine Rolle mehr gespielt hatten - eben die des ,Geistes‘ sowie die der ,Sprache‘ als abstraktes, aber reales Gesamtgebilde einer Sprachgemeinschaft.

Vergleicht man nun das Verfahren der Sprachinhaltsforscher mit Grimms Philologie, fallen in der Tat wesentliche Gemeinsamkeiten auf. Zum einen sind wie bei Grimm auch hier die Grenzen zwischen den sprachwissenschaftlichen Gattungen fließend, denn schon von ihrem Grundansatz her lehnt die Sprachinhaltsforschung jede Trennung einer Formlehre von der Behandlung der Wort- und Sprachinhalte ab. Das zeigt sich exemplarisch an Weisgerbers „inhaltbezogener Grammatik“, die er der „lautbezogenen“ seiner Vorgänger gegenüberstellt. $^{30}$ Hier wird etwa die herkömmliche Unterscheidung zwischen Singular und Plural, die sich an den Wortformen festmacht, im Hinblick auf die komplexeren Verhältnisse der Sprachinhalte revidiert: Bei einem singularium tantum wie ,Gold', das keinen Plural bildet, kann von ,Singular' nicht die Rede sein (,Wo kein Plural ist, kann auch kein Singular sein“), ${ }^{31}$ doppelte Plurale wie ,Wort/Wörter' und ,Wort/Worte' rühren daher, dass eine Mehrzahl entweder als

28 Arens 1969: 361.

29 Vgl. zu dieser ganzen Entwicklung und Weisgerbers Stellenwert darin Knobloch 2000. Die antipositivistische Welle setzt natürlich noch früher ein, in der Romanistik etwa mit Karl Vossler und seiner Schule, vgl. Knobloch 2005: 63-86.

30 Vgl. etwa Arens 1969: 542; Weisgerber 1953: 97.

31 Weisgerber 1963: 78. 
Vielheit (Wörter) oder als Kollektiv (Worte) gesehen werden kann. ${ }^{32}$ Die reine Formbeschreibung greift zu kurz, solange man nicht zugleich die Inhalte berücksichtigt - in diesem Falle etwa, dass und warum es der deutschen Sprache an dieser besonderen ,inhaltlichen' Stelle ihrer Semantik wichtig erscheint, nicht nur zwischen Singular und Plural, sondern zwischen Vielheit und Kollektiv zu unterscheiden. „[E]s wird [...] die Fragwürdigkeit mancher in der Formenlehre üblicher Fachausdrücke sichtbar, ebenso die Unvollständigkeit der vorwiegend auf Endungen gestützten Bestandsaufnahme der Formenkreise. “33 Ebenso wenig wie bei Grimm also beschränkt sich die Grammatik der Sprachinhaltsforscher auf die Form; auch sie versenkt sich gleichsam in den Stoff, in den Inhalt der Wörter.

$\mathrm{Zu}$ dieser ,ganzheitlichen Sprachbetrachtung‘ gehört nun auch, und das ist der zweite Punkt, der uns an Grimm denken lässt, dass sie jeden Versuch, eine Fachsprache von der Gemeinsprache abzulösen, als verfehlt betrachtet wobei dies nun, anders als bei Grimm, zu einem explizit reflektierten Grundsatz des neuen Ansatzes erklärt wird. Der Satz ,ich verstehe darunter das und das ‘ - der konstitutive Satz einer jeden Fachsprache - sei, so der Grundgedanke, nur von einer gemeinen oder Volkssprache aus verständlich. Die Fachsprache fuße stets auf dem gemeinen Sprachgebrauch und führe letztlich immer auf ihn zurück. Das ist die Konsequenz des von Weisgerber und seiner Schule sogenannten ,muttersprachlichen Apriori‘: des Gesetzes, dass man niemals hinter die eigene Muttersprache zurückkönne, da alles später Versprachlichte letztlich an sie zurückgebunden werden muss. ${ }^{34}$ In der irrigen Meinung, sie könnten durch willkürliche Begriffsbestimmungen gleichsam aus den einzelnen Volks- oder Muttersprachen herauskommen und den Dingen auf objektive Weise näherkommen, verkennen die Fachterminologien, so Weisgerbers Fundamentalkritik an den Wissenschaftssprachen, dass die Dinge immer nur durch die Vermittlung der Sprache zugänglich sind. Nicht die Dinge, wie sie an sich sind, sondern nur ganz bestimmte „Sehweisen“ der Dinge bringen die Fachsprachen zum Ausdruck - Sehweisen, die „dem Gesetz der Sprachverschiedenheit unterliegen, also die Abhängigkeit von einer Muttersprache nie ganz überwinden können“. ${ }^{35}$ Insbesondere für die Sprachwissenschaft gälte somit das Prinzip, dass die Fachsprache sich ihre Rückbindung an die Objektsprache stets vor Augen halten soll.

32 Vgl. ebd.

33 Weisgerber 1953: 208.

34 Vgl. etwa Gipper 1971.

35 Weisgerber 1957: 246. 
Schaut man sich nun ihre eigene Wissenschaftsprosa an, drängt sich tatsächlich der Eindruck auf, dass bei den Sprachinhaltsforschern nicht weniger als bei Grimm Meta- und Objektsprache in eins fallen. Das ist besonders bei Jost Trier der Fall, der als Initiator der Wortfeldtheorie bekannt wurde. Unter ,Wortfeld“ versteht Trier eine Gruppe inhaltlich eng benachbarter Wörter, die sich „vermöge Interdependenz ihre Leistungen gegenseitig zuweisen“. ${ }^{36}$ Die Bedeutung der einzelnen Wörter eines Wortfeldes - Beispiele sind Farbwörter, Verwandtschaftsbezeichnungen, Schulnoten - ergibt sich nur aus ihrer Differenz zueinander. Dies lässt natürlich an Saussure denken, allerdings geht Trier insofern eigene Wege, als es ihm von Anfang an um diachrone Prozesse geht: um das ,Werden“ der Differenzen in dem prinzipiell offenen, also nicht als System eingrenzbaren Bereich des Wortschatzes. ${ }^{37}$ So beruft sich Trier nicht auf Saussure, sondern auf Goethe, dem es in seinen ,morphologischen Heften“ ja darum zu tun war, „das Simultane und zugleich das Sukzessive zu sehen“. ${ }^{38}$ In dem Begriff des ,Feldes“, der auf Trier „wie eine plötzliche Erleuchtung“39 gewirkt habe, als er ihm bei dem Soziologen Gunther Ipsen begegnete, sah er ein Mittel zur Lösung der Aufgabe, Synchrones und Diachrones zusammenzudenken. Allerdings ist „Begriff‘ nicht das richtige Wort: denn Triers Schlüsselwort ,Feld“ ist vielmehr eine Metapher - ein Umstand, den Trier selbst reflektiert und der sich für seine Wortfeldtheorie als entscheidend erweisen sollte. Denn die Entwicklung von Triers Theorie gestaltet sich nicht zuletzt als die über Jahrzehnte anhaltende Suche nach einem geeigneten Bild für das Gemeinte. So heißt es zunächst, das Wortfeld sei „mosaikartig“, ${ }^{40}$ was dann aber später als zu statisch verworfen wird. Statt vom Mosaik ist dann vom Pferderennen die Rede „das Feld der Pferde, die in Raum und Zeit zum Ziel rennen und zwischen denen die gegenseitigen Stellungsverhältnisse und damit Stellenwerte sich unaufhörlich verschieben“. ${ }^{41}$ Im Bild des Pferderennens komme „das Dynamische, das Moment des Wandels und des Werdens“ besser zur Geltung. ${ }^{42}$ Aber damit nicht genug: Noch 1968, zwei Jahre vor seinem Tod, ist Trier damit beschäftigt, das Bild, auf dem seine Theorie beruht, umzugestalten. Statt Pferden kommen jetzt Sterne ins Spiel:

\footnotetext{
36 Trier 1973: 189.

37 Vgl. ebd.

38 Ebd.: 193. Zu Goethes Morphologie vgl. Geulen 2016.

39 Trier 1973: 189.

40 Ebd.: 55.

41 Ebd.: 193. Vgl. dazu Peil 1998: 144.

42 Trier 1973: 193.
} 
Die Sprachen arbeiten [...] mit injunktiven Wortinhalten (Ingriffen) nicht mit disjunktiven Inhalten (Umgriffen). Das Bild der scharf sich aneinanderlegenden Wortumrandungen ist daher zu ersetzen durch ein Miteinander sternförmig ausstrahlender Kerne, die so zueinander liegen, daß die äußersten Strahlenspitzen eines Kerns zwischen die Strahlenspitzen der benachbarten Kerne eingreifen oder eingreifen können. Der Gedanke der wechselseitigen inhaltlichen Abhängigkeit der Glieder im Feld bleibt auch nach der Korrektur ganz in seinem Recht. ${ }^{43}$

Darüber schreibt später Weisgerbers Schüler Helmut Gipper: „die Ausdrücke injunktiv und disjunktiv und die bildreiche Umschreibung sind doch schwer nachzuvollziehen. Disjunktiv im Sinn von ,ausschließend‘ mag noch hingehen, aber die Opposition injunktiv, die ich in keinem Fachwörterbuch der Linguistik oder der Logik gefunden habe, bleibt unklar““ ${ }^{44}$ In der Tat scheint der Terminus ,Ingriff“ eine Art Hapaxlegomenon zu sein: nicht einmal Google kennt ihn. Ob er deswegen so unverständlich ist, wie Gipper meint, ist indessen fraglich. Eher könnte man der Metaphorik an dieser Stelle vorwerfen, sie sei geradezu überdeutlich konstruiert. Dass die Elemente eines Wortfeldes nicht Begriffe oder fest umrissene Umgriffe, sondern eben Ingriffe sind, will offenbar sagen, dass in jedem dieser Elemente stets andere, gleichsam hineinragende Elemente mitwirken. Klar ist auch, dass sich Triers ,Fachterminologie an dieser Stelle von den Eigenschaften der durch sie beschriebenen Objektsprache inspirieren lässt: ,Begriff‘, ,Umgriff“ und ,Ingriff" sind offenbar selbst ein Wortfeld. Meta- und Objektsprache gehen, wie es scheint, ein inniges Verhältnis ein.

Bei näherem Hinsehen sind allerdings Zweifel angezeigt. Sicher, ,Ingriff' ist eine metaphorische Neubildung, die, wie es scheint, in der Gemeinsprache wurzelt - nämlich in dem Wortfeld der mit ,-griff' gebildeten Substantive (,Begriff', ,Umgriff'). So wie Grimms ,stark‘ oder ,schwach` verhält sich dieser Fachbegriff letztlich aber doch nicht. Nicht nur bleibt er morphologisch unfruchtbar - es bilden sich keine abgeleiteten Formen wie etwa ,ingreifen', so wie ,Ablaut' bei Grimm zu ,ablauten' führt oder ,schwach` zu ,schwächen`-, sondern er entsteht auch eher durch ein Wortspiel als durch sinnlich erfassbare metaphorische Wortbildung. Hier wird auf morphologischer Ebene mit dem bereits zum Terminus erstarrten Wort ,Begriff“ gespielt - und gerade nicht ein sonst nicht Erfassbares bildlich zum Ausdruck gebracht. Bei Grimm gewinnt man den Eindruck, die Fachtermini entstehen ebenso spontan, wie überhaupt in einer Sprache Wörter fortwährend umgeformt und neu geprägt werden: im Zuge sich neu formierender Gedanken und Erkenntnisse, für die es noch keinen passenden Ausdruck gibt. Dagegen wirken Triers Neologismen planvoll - eben wie Teile einer Fachsprache,

43 Ebd.: 195.

44 Gipper 1998: 39. 
um deren Neuerfindung es ihm zu tun ist. Dass ein Wort wie ,Ingriff‘ deutsch aussieht, dass es aus deutschem Wortbestand gebildet wird - wobei man dabei bedenken muss, dass das Ganze auf Lehnübertragungen aus dem Lateinischen beruht (conceptus, von capere: ,greifen') -, vermag nicht darüber hinwegzutäuschen, dass an dieser Stelle ein Neologismus genauso ,willkürlich“ geprägt und definiert wird, wie es bei jedem technischen, eigens eingeführten Fremdwort der Fall ist. Nur weil eine Metasprache sich bewusst metaphorisch gestaltet, heißt das nicht, dass sie mit ihrer Objektsprache zusammenfällt. ${ }^{45}$

Hinzu kommt aber ein noch bedeutenderer Unterschied. Die Theorien der Sprachinhaltsforscher entfalten sich nämlich auf der Basis eines Sprachdenkens, das ,Sprache“ stets als die Sprache einer ,Sprachgemeinschaft' versteht: als Sprache eines Volks oder als ,Muttersprache‘. Auch Grimm redet von ,Volk‘ und von ,Muttersprache‘, aber bei Trier und Weisgerber erhalten diese Bezeichnungen ein anderes Gewicht. Der Sprachinhalt, um den es den Sprachinhaltsforschern geht, ist stets der Inhalt einer ganz bestimmten Sprache (etwa: Deutsch oder Keltisch); und alles Reden, alle parole, vollzieht sich stets innerhalb der Grenzen einer Sprache, deren feste Umrisse durch die ebenso feste Identität der sie besitzenden ,Sprachgemeinschaft' gegeben sind. Triers Analysen der Bedeutungsnuancen der gegenseitig aufeinander wirkenden ,injunktiven' Wörter eines Wortfeldes setzen eine äußerst disjunktive Idee von ,Sprachen“ voraus: jede Sprachverwendung ist stets entweder Deutsch oder Englisch oder Latein; sie kann nicht außerhalb dieser sich ausschließenden ,Sprachen“ existieren und sie kann nicht zugleich mehreren Sprachen zugeordnet werden. Weisgerber erhebt es gar zur Pflicht, die Wörter stets genauso zu verwenden, wie der gemeine Gebrauch sie festlegt:

Wenn in der Sprache eines Volkes [...] die Erfahrungen der früheren Geschlechter ihren Ausdruck gefunden haben, so ist es Pflicht der jeweiligen Träger einer Sprache, dieses Gut zu bewahren, solange es nicht durch neue Erfahrungen und Erkenntnisse überholt ist. ${ }^{46}$

Gewiss: sowohl Trier als auch Weisgerber führen wie Grimm Neuschöpfungen ein - bei Trier nicht nur in der Rede vom ,Ingriff‘, sondern auch etwa dort, wo es heißt, ein jedes Wort ,ergliedere' sich aus dem Ganzen des Wortschatzes, während dieser sich wiederum in die einzelnen Wörter ,ausgliedere ${ }^{47}$ oder wo

45 Ferner macht Trier selbst deutlich, dass er auf die Festlegung einer in ihrer Bedeutung stabilen, nicht-metaphorischen Bedeutung zielt: „Wir vertrauen darauf, daß es der Metapher Feld so ergeht wie allen Metaphern, daß sie schließlich verblaßt, daß schließlich niemand mehr an die Herkunft, sondern alle nur an das denken, was wir unter der Fahne Feld wirklich tun.“ (Trier 1973: 195f.)

46 Weisgerber 1929: 130.

47 Trier 1973: 147. 
es bei Weisgerber um das ,Worten“ der Welt geht. ${ }^{48}$ Aber all diese Neologismen werden als Neologismen so deutlich ausgestellt, dass sie die Identität der Muttersprache, in der sie bestenfalls eine Insel bilden, an keiner Stelle infrage stellen im Gegenteil. Wie Fremdwörter markieren sie gerade die Grenzen der heimischen Sprache, indem sie sie mit großer Geste überschreiten. Und auch innerhalb dieser Grenzen - überall dort, wo keine solche als Fachwörter ausgewiesenen Ausdrücke verwendet werden - ist die Prosa der Sprachinhaltsforscher, mit der von Grimm verglichen, ungleich einsprachiger, ich möchte sagen: muttersprachlicher. Wo nicht ausdrücklich in einer als fremd markierten Fachsprache geredet wird, halten sich Trier und Weisgerber genau an das Idiomatische der deutschen Gegenwartssprache; man spürt regelrecht die peinliche Sorge, mit der sie jede noch so kleine Abweichung vom etablierten Wortgebrauch vermeiden. Bei Weisgerber ist jede Substantiv-Verb-Verbindung vertraut, die Adjektive passen wie angegossen, kein Wort fällt auf. Etwas wird mit etwas „in Einklang gebracht“; ${ }^{49}$ ein Ideal ist „nie erreichbar“; ${ }^{50}$ in den Gegenstand vertieft man sich „eindringlich““. ${ }^{51}$ Es ist die Sprache des Duden-Stilwörterbuchs.

Ganz anders, wie wir gesehen haben, die Prosa Jacob Grimms. Von der Orthographie über den Wortgebrauch bis in die Syntax hinein ist Grimms Sprache alles andere als dem ,gemeinen Sprachgebrauch“ gemäß. Vor allem aber ist sie mehrsprachiger - wobei dieser Begriff den Sachverhalt nicht recht trifft. ,Mehrsprachig' setzt nämlich diskrete Einzelsprachen voraus, die dann kombiniert oder vermischt werden. Bei Grimm aber sind die Sprachen alles andere als ,disjunktiv، abgegrenzt. Grimms sprachwissenschaftliche Prosa ist strenggenommen weder mehrsprachig noch einsprachig, sie ist vielmehr in nur sehr geringem Grad überhaupt ,sprachig، (im Sinne von ,einer Einzelsprache zugehörig'). ${ }^{52}$ Man schlage irgendeine beliebige Seite der Deutschen Grammatik, der Geschichte der deutschen Sprache oder des Deutschen Wörterbuchs auf und man wird mit einem Schriftbild konfrontiert, das bunter kaum sein könnte: Was Neuhochdeutsch, was Latein, was ein Beleg aus einer vergangenen germanischen Sprache ist, lässt sich zunächst kaum auseinanderhalten, da die verschiedenen Register in der Regel nicht typographisch voneinander abgehoben werden:

48 Vgl. Weisgerber 1955.

49 Weisgerber 1953: 9.

50 Ebd.

51 Ebd.: 10.

52 Der Begriff der ,Sprachigkeit‘ geht auf Robert Stockhammer zurück, vgl. die Einleitung zu Arndt et al. 2007: 22-26. Zum Gebrauch des Terminus im hier gemeinten Sinne vgl. Martyn 2014. 
Genug von den vocalen der goth. schwachen flexion; wichtiger ist es auf das kennzeichen $\mathrm{N}$ zu achten [.. .]. von aba maritus wird nicht abam sondern abnam, von vatô aqua, namô nomen nicht vatam namam, sondern vatnam namnam gebildet, wobei freilich auch der vorstehende vocal schwindet, denn es sollte heiszen abanam vatanam namanam. ${ }^{53}$

Im Deutschen Wörterbuch wird zwar sowohl in Antiqua wie auch kursiv gesetzt, allerdings ohne dass dies zur Klärung der Lage beitrüge. Hier ein Auszug aus dem berühmten Artikel zum Adjektiv ,arm‘:

arm, miser, pauper, elend und dürftig, goth. arms, ahd. aram, mhd. arm, alts. arm, mnl. arem, aerm, nnl. arm, ags. earm, fries. erm, altn. armr, schw. dän. arm, also gleich dem vorausgehenden subst. allen deutschen sprachen gemein [...]. armen hiesz amplecti, in manus tollere, umarmen, das grenzt geradezu an erbarmen, bemitleiden; wie gefühlvoll erschiene die sprache, welcher der arme ein solcher ist, den man mitleidig, liebreich aufnimmt und in die arme schlieszt. (DWB 1: $553 \mathrm{f.}$ )

Das Kursivgedruckte ist ununterschieden Deutsch und Latein, gibt sowohl Wortgeschichte als auch Bedeutungen wieder - alles, was nicht Beleg ist. Aber auch diese Trennung wird nicht durchgehend eingehalten. Das vorletzte, in Antiqua gesetzte Wort in diesem Zitat gehört gleichermaßen zur neuhochdeutschen Sprache der Erklärung als auch, sofern die hier von Grimm vorgeschlagene Etymologie zutrifft, zu den germanischen Sprachen der Belege - denn nur in der älteren Sprache, nicht in der neueren, sind ,Arme“ die zum mitleidigen Aufheben der ,Armen` bestimmten Körperteile. In diesem Gebrauch des in seiner etymologischen Tiefe neuergründeten Worts hört jede Unterscheidung zwischen Meta- und Objektsprache, zwischen der Sprache des Lexikographen und der seiner Belege auf.

Grimm ist sich darüber im Klaren, wie sehr er mit diesem ebenso gelehrten wie wilden Gemisch die Leser des Deutschen Wörterbuchs überfordert. Bezeichnenderweise sah er darin allerdings kein Problem:

Auch ist gar nicht noth, dasz allen alles verständlich, dasz jedem jedes wort erklärt sei, er gehe an dem unverstandnen vorüber und wird es das nächstemal vielleicht fassen. nenne man ein gutes buch, dessen verständnis leicht wäre und nicht einen unergründlichen hintergrund hätte. das wörterbuch insgemein führt so schweren stof mit sich, dasz die gelehrtesten bei manchem verstummen oder noch nicht rechten bescheid wissen. [...] leser jedes standes und alters sollen auf den unabsehbaren strecken der sprache nach bienenweise nur in die kräuter und blumen sich niederlassen, zu denen ihr hang sie führt und die ihnen behagen. (DWB 1: xii)

Grimm ist bewusst, dass seine Leser ihn nicht verstehen werden. Die Sprache, die er mit ihnen teilt, ist nicht von der Art, dass sie von allen verstanden wird.

53 Grimm 1880/1999: 655. 
Dabei geht es aber nicht nur um die Zweiteilung von Fach- und Gemeinsprache - sondern um eine sprachliche Vielfalt, die sich überall und zwischen allen Ständen und Lesern verbreitet.

\section{Schluss}

Was kann es heißen, Wissenschaft auf Deutsch zu betreiben? Unter welchen Bedingungen ist dies möglich? Und in welchem Sinne muss man ,Wissenschaft ${ }^{\text {* }}$ und ,Deutsch“ verstehen, damit von ,Deutsch als Wissenschaftssprache“ sinnvoll gesprochen werden kann?

Sofern unsere beiden Fälle als beispielhaft gelten dürfen, hat sich im Hinblick auf diese Fragen zweierlei gezeigt. Erstens, dass der Versuch, Wissenschaft auf Deutsch als Muttersprache zu betreiben, misslingen muss. Die Fachsprache, die Trier entwickelt, sieht zwar ,deutsch` aus, steht aber zur Gemeinsprache in genau demselben Verhältnis, wie es jede Fachterminologie tut - auch eine aus lateinischen oder griechischen Fremdwörtern bestehende. Anders kann es nicht sein, wenn Wissenschaft per definitionem mit der Objektivierung ihres Gegenstands einhergeht. Denn die Kluft zwischen Erkenntnissubjekt und Gegenstand verlangt gerade, zumindest in der Sprachwissenschaft, dass das Subjekt seine Erkenntnisse in einer anderen Sprache festhält als in der, die es erforscht. In der Sprachwissenschaft wird man dem Anspruch auf Objektivierung nur dann gerecht, wenn man die Gemeinsprache hinter sich lässt.

Bei Grimm nun liegt die Sache offenbar anders. Denn seine Wissenschaft ist keine, die eine objektivierende Distanz zu ihrem Gegenstand voraussetzt; und die Sprache, in der Grimm sie betreibt, ist nicht ,Muttersprache“ im Sinne von Weisgerber. Wenn Grimms Wissenschaftssprache ,Deutsch“ ist, und er selber würde dies sicherlich so sehen, dann handelt es sich um ein ganz anderes ,Deutsch“ als das von Trier und Weisgerber. Denn die Grenzen von Grimms Deutsch sind so ausgedehnt, flüssig und diffus, dass von einer ,Sprachgemeinschaft‘, deren Besitz es wäre, überhaupt nicht die Rede sein kann. Es gehört allen und niemandem. ${ }^{54}$ Bei Grimm wird also tatsächlich ,Wissenschaft` auf ,Deutsch' betrieben - aber es ist eine Wissenschaft, und dies ist das zweite, was sich hier gezeigt hat, die dem Gesetz der Objektivierung nicht folgt; und es ist

54 In seiner Studie zur Geschichte der Wissenschaftssprachen geht Michael Gordin davon aus, es sei ein „truism“, dass „scientific activity is communicated in a language“ - „in a particular, specific language, shared by a community of speakers“ (Gordin 2015: 4). Der Fall Grimm legt nahe, dass diese Annahme alles andere als selbstverständlich ist. 
ein Deutsch, das die Grenzen dessen sprengt, was unter dem Begriff einer ,Sprache' wie dem Deutschen gemeinhin verstanden wird. Grimm macht vor, was es heißt, Wissenschaft auf Deutsch zu betreiben - und was es heißt, beide Begriffe neu zur Disposition zu stellen.

\section{Literatur}

Andresen, Karl Gustav (1968): Über die Sprache Jacob Grimms. Reprographischer Nachdruck der Erstausgabe Leipzig 1869. Wiesbaden: Sändig.

Arens, Hans (1969): Sprachwissenschaft. Der Gang ihrer Entwicklung von der Antike bis zur Gegenwart, 2. Aufl. Freiburg i. Br., München: Alber.

Arndt, Susan, Dirk Naguschewski \& Robert Stockhammer (2007): Einleitung. In: Dies. (Hrsg.), Exophonie. Anders-Sprachigkeit (in) der Literatur. Berlin: Kadmos, 7-30.

Foucault, Michel (1971): Die Ordnung der Dinge. Eine Archäologie der Humanwissenschaften. Frankfurt a.M.: Suhrkamp.

Geulen, Eva (2016): Aus dem Leben der Form. Goethes Morphologie und die Nager. Berlin: August.

Ginschel, Gunhild (1956): Jacob Grimm - Aufgaben und Probleme der Exzerption. In: Rolf Hiersche, Erika Ising \& Gunhild Ginschel (Hrsg.), Aus der Arbeit zu einem historischen Wörterbuch der sprachwissenschaftlichen Terminologie. Berlin: Akademie, 47-110.

Gipper, Helmut (1971): Das Sprachapriori. Sprache als Voraussetzung menschlichen Denkens und Erkennens. Stuttgart-Bad Cannstatt: Frommann-Holzboog.

Gipper, Helmut (1998): Das sprachliche Feld in Jost Triers und in heutiger Sicht. In: Werner Zillig (Hrsg.), Über Jost Trier. Münster: Aa, 28-53.

Gordin, Michael D. (2015): Scientific Babel. The Language of Science from the Fall of Latin to the Rise of English. Chicago, London: University of Chicago Press.

Grimm, Jacob \& Wilhelm Grimm (1854-1971): Deutsches Wörterbuch. 33 Bde. Leipzig: Hirzel (= DWB).

Grimm, Jacob (1864): Über das Pedantische in der deutschen Sprache. In: Ders., Reden und Abhandlungen. Berlin: Dümmler, 327-373.

Grimm, Jacob (1870/1989): Deutsche Grammatik 1. In: Jacob Grimm \& Wilhelm Grimm: Werke. Forschungsausgabe. Abt. 1, Bd. 10. Hrsg. von Elisabeth Feldbusch \& Ludwig Erich Schmitt. Besorgt durch Wilhelm Scherer. 2. Nachdruck der Ausgabe Berlin, Dümmler 1870. Hildesheim u. a.: Olms-Weidmann.

Grimm, Jacob (1878/1989): Deutsche Grammatik 2. In: Jacob Grimm \& Wilhelm Grimm: Werke. Forschungsausgabe. Abt. 1, Bd. 11. Hrsg. von Elisabeth Feldbusch \& Ludwig Erich Schmitt. Besorgt durch Wilhelm Scherer. 2. Nachdruck der Ausgabe Berlin, Dümmler 1878. Hildesheim u. a.: Olms-Weidmann.

Grimm, Jacob (1819/1995): Deutsche Grammatik 1. In: Jacob Grimm \& Wilhelm Grimm: Werke. Forschungsausgabe. Abt. 1, Bd. 9,1. Hrsg. von Elisabeth Feldbusch \& Ludwig Erich Schmitt. Reprographischer Nachdruck der Ausgabe Göttingen 1819. Hildesheim u. a.: Olms-Weidmann.

Grimm, Jacob (1840/1995): Deutsche Grammatik 1. In: Jacob Grimm \& Wilhelm Grimm: Werke. Forschungsausgabe. Abt. 1, Bd. 9,2. Hrsg. von Elisabeth Feldbusch \& Ludwig Erich 
Schmitt. Reprographischer Nachdruck der Ausgabe Göttingen 1840. Hildesheim u. a.: Olms-Weidmann.

Grimm, Jacob (1880/1999): Geschichte der deutschen Sprache 2. In: Jacob Grimm \& Wilhelm Grimm: Werke. Forschungsausgabe. Abt. 1, Bd. 16. Nach der Ausgabe von Hermann Grimm neu hrsg. von Elisabeth Feldbusch. Mit einer Einleitung von Maria Herrlich. 2. Nachdruck der 4. Aufl. Leipzig, Hirzel 1880. Hildesheim u. a.: Olms-Weidmann. Herrlich, Maria (1998): Organismuskonzept und Sprachgeschichtsschreibung. Die Geschichte der deutschen Sprache von Jacob Grimm. Hildesheim u. a.: Olms-Weidmann.

Ising, Erika (1956): Die Begriffe ,Umlaut‘ und ,Ablaut‘ in der Terminologie der frühen deutschsprachigen Grammatik. In: Rolf Hiersche, Erika Ising \& Gunhild Ginschel (Hrsg.), Aus der Arbeit zu einem historischen Wörterbuch der sprachwissenschaftlichen Terminologie. Berlin: Akademie, 21-46.

Knobloch, Clemens (2000): Begriffspolitik und Wissenschaftsrhetorik bei Leo Weisgerber. In: Klaus D. Dutz (Hrsg.), Interpretation und Re-Interpretation. Aus Anlaß des 100. Geburtstages von Johann Leo Weisgerber (1899-1985). Münster: Nodus, 145-174.

Knobloch, Clemens (2005): Volkhafte Sprachforschung. Studien zum Umbau der Sprachwissenschaft in Deutschland zwischen 1918 und 1945. Tübingen: Niemeyer.

Krapf, Veronika (1993): Sprache als Organismus. Metaphern - Ein Schlüssel zu Jacob Grimms Sprachauffassung. Kassel: Schriften der Brüder-Grimm-Gesellschaft.

Martyn, David (2014): Es gab keine Mehrsprachigkeit, bevor es nicht Einsprachigkeit gab. Ansätze zu einer Archäologie der Sprachigkeit (Herder, Luther, Tawada). In: Till Dembeck \& Georg Mein (Hrsg.), Philologie und Mehrsprachigkeit. Heidelberg: Winter, 39-52.

Peil, Dietmar (1998): Jost Trier und die Metaphorik. In: Werner Zillig (Hrsg.), Über Jost Trier. Münster: Aa, 129-153.

Pörksen, Uwe (1986): Deutsche Naturwissenschaftssprachen. Historische und kritische Studien. Tübingen: Narr.

Pörksen, Uwe (1994): Wissenschaftssprache und Sprachkritik. Untersuchungen zu Geschichte und Gegenwart. Tübingen: Narr.

Reichmann, Oskar (1991): Zum Urbegriff in den Bedeutungserläuterungen Jacob Grimms (auch im Unterschied zur Bedeutungsdefinition bei Daniel Sanders). In: Alan Kirkness et al. (Hrsg.), Studien zum Deutschen Wörterbuch von Jacob Grimm und Wilhelm Grimm. Bd. 1. Tübingen: Niemeyer, 299-345.

Rheinberger, Hans-Jörg (1997): Toward a History of Epistemic Things. Synthesizing Proteins in the Test Tube. Stanford: Stanford University Press.

Simon, Ernst (1929): Zu Jacob Grimms Sprache, Stil und Persönlichkeit. In: Deutsche Vierteljahrsschrift für Literaturwissenschaft und Geistesgeschichte 7, 515-559.

Stukenbrock, Anja (2005): Sprachnationalismus. Sprachreflexion als Medium kollektiver Identitätsstiftung in Deutschland (1617-1945). Berlin, New York: De Gruyter.

Trier, Jost (1973): Aufsätze und Vorträge zur Wortfeldtheorie. Hg. von Anthony van der Lee \& Oskar Reichmann. Den Haag, Paris: Mouton.

Weisgerber, Leo (1929): Muttersprache und Geistesbildung. Göttingen: Vandenhoeck \& Ruprecht.

Weisgerber, Leo (1953): Vom Weltbild der deutschen Sprache. In: Ders., Von den Kräften der deutschen Sprache. Bd. II.1, 1. Halbbd.: Die inhaltbezogene Grammatik. Düsseldorf: Schwann.

Weisgerber, Leo (1955): Das Worten der Welt als sprachliche Aufgabe der Menschheit. In: Sprachforum 1 (1), 10-19. 
Weisgerber, Leo (1957): Die Muttersprache im Aufbau unserer Kultur. In: Ders., Von den Kräften der deutschen Sprache. Bd. III. 2. Aufl. Düsseldorf: Schwann.

Weisgerber, Leo (1963): Die vier Stufen in der Erforschung der Sprachen. Düsseldorf: Schwann.

Wyss, Ulrich (1979): Die wilde Philologie. Jacob Grimm und der Historismus. München: C.H. Beck. 